\title{
Unruptured aneurysms in the elderly: perioperative outcomes and cost analysis of endovascular coiling and surgical clipping
}

\author{
Nicole A. Silva, BS, ${ }^{1}$ Belinda Shao, BA, ${ }^{1}$ Michael J. Sylvester, MD, ${ }^{2}$ Jean Anderson Eloy, MD, $, 1,3,4$ \\ and Chirag D. Gandhi, MD'
}

1Department of Neurological Surgery and 2Department of Otolaryngology, Rutgers New Jersey Medical School, Newark, New Jersey; ${ }^{3}$ Department of Otolaryngology, University of Michigan, Ann Arbor, Michigan; and ${ }^{4}$ Department of Neurological Surgery, Westchester Medical Center/New York Medical College, Valhalla, New York

OBJECTIVE Observation and neurosurgical intervention for unruptured intracranial aneurysms (UIAs) in the elderly population is rapidly increasing. Cerebral aneurysm coiling (CACO) is favored over cerebral aneurysm clipping (CAC) in elderly patients, yet some elderly individuals still undergo CAC. The cost-effectiveness of treating UIAs requires further exploration. Understanding the effect of intervention on hospital charges and length of stay (LOS) as well as perioperative mortality and complications can further shed light on its economic impact. The purpose of this study was to analyze the cost and perioperative outcomes of UIAs in elderly patients ( $\geq 65$ years of age) after CACo or CAC intervention.

METHODS Retrospective cohorts of CACo and CAC admissions were extracted from National (Nationwide) Inpatient Sample data obtained between 2002 and 2013, forming parallel intervention groups to compare the following outcomes between elderly and nonelderly patients: average LOS and mean hospital admission costs, in-hospital mortality, and complications. Covariates included sex, race or ethnicity, and comorbidities.

RESULTS Elderly patients undergoing CAC experienced an average LOS of 8.0 days, whereas elderly patients undergoing CACo stayed an average of 3.2 days. The mean hospital charges incurred during admission totaled $\$ 95,960$ in the elderly patients who underwent CAC versus $\$ 87,960$ in the ones who underwent CACo. Elderly patients in whom CAC was performed had a $2.2 \%$ rate of in-hospital mortality, with a 2.6 greater adjusted odds of in-hospital mortality than nonelderly patients treated with CAC. In contrast, elderly patients who underwent CACo had a 1.36 greater adjusted odds of in-hospital mortality than their nonelderly counterparts. Compared to nonelderly patients receiving both interventions, elderly individuals had a significantly higher prevalence of various comorbidities and incidence of complications. Elderly patients who received CAC experienced a $10.3 \%$ incidence rate of perioperative stroke, whereas their CACo counterparts experienced this complication at a rate of $3.5 \%$. Elderly patients treated with CAC had greater odds of perioperative acute renal failure, whereas their CACo counterparts had greater odds of perioperative deep venous thrombosis and pulmonary embolism.

CONCLUSIONS Intervention with CAC and CACo in the elderly is resource intensive and is associated with higher risk than in the nonelderly. Those deciding between intervention and conservative management should consider these risks and costs, especially the $2.2 \%$ postoperative mortality rate associated with CAC in the elderly population. Further comparative cost-effectiveness research is needed to weigh these costs and outcomes against those of conservative management.

https://thejns.org/doi/abs/10.3171/2018.1.FOCUS17714

KEYWORDS elderly; cerebral aneurysm coiling; cerebral aneurysm clipping; endovascular coiling; surgical clipping; unruptured intracranial aneurysm; cost analysis

ABBREVIATIONS ARF = acute renal failure; $\mathrm{CAC}=$ cerebral aneurysm clipping; $\mathrm{CACo}=$ cerebral aneurysm coiling; $\mathrm{CHF}=$ congestive heart failure; $\mathrm{COPD}=$ chronic obstructive pulmonary disease; DVT = deep venous thrombosis; ICD-9 = International Classification of Diseases, Ninth Revision; LOS = length of stay; NIS = National (Nationwide) Inpatient Sample; PE = pulmonary embolism; PVD = peripheral vascular disorder; $\mathrm{SAH}=$ subarachnoid hemorrhage; UIA = unruptured intracranial aneurysm. ACCOMPANYING EDITORIAL DOI: 10.3171/2018.2.FOCUS1871.

SUBMITTED November 27, 2017. ACCEPTED January 18, 2018.

INCLUDE WHEN CITING DOI: 10.3171/2018.1.FOCUS17714. 
$\mathrm{U}$ NRUPTURED intracranial aneurysms (UIAs) carry a risk of rupture, which may lead to intracranial stroke, subarachnoid hemorrhage $(\mathrm{SAH})$, coma, and death.4,6,7,9,15,25,32 Current management options include intervention with cerebral aneurysm coiling (CACo), cerebral aneurysm clipping (CAC), and observation of growth with periodic imaging. ${ }^{15,19,32}$ The decision to intervene in the course of a UIA is made by weighing an individual patient's lifetime risk for rupture against the risks associated with CAC or CACo intervention. ${ }^{11,32}$ Risk factors for rupture include number of aneurysms, size, location, and morphology as well as history of SAH, increased age, hypertension, ethnic background, smoking, and relevant family history., ${ }^{7,23-25,32}$ Lifetime risk of rupture also considers a patient's life expectancy. ${ }^{32}$ As life expectancy continues to rise in the elderly, there is higher risk of rupture and SAH. ${ }^{29}$ Likewise, intervention-related complications associated with age and its accompanying comorbidities complicate the management of elderly patients both intraoperatively and postoperatively. . $^{3,11,15,19,28,31,32}$

In the past decade, the number of surgical and endovascular interventions performed to treat unruptured aneurysms has significantly increased. This change has been driven by rapidly evolving and safer treatment modalities as well as concurrent improvements and broad adoption of diagnostic imaging techniques. ${ }^{7,8}$ Specifically in 2010, there were 1.2 million neurosurgical procedures performed, a number that continues to rise. ${ }^{33}$ With more than $\$ 4.4$ trillion projected to be spent on health care in the US in 2018 , it is necessary to quantify cost-effectiveness and health outcomes for unruptured aneurysms in the elderly population. ${ }^{14,17,33}$ As the baby boomers' aging population expands worldwide and detection of unruptured aneurysms is on the rise, ${ }^{15}$ the need for economically favorable management of unruptured aneurysms in the elderly becomes increasingly relevant. ${ }^{32}$

Results from the landmark International Subarachnoid Aneurysm Trial in 2002 and 2015 assessed outcomes of intervention in ruptured aneurysms only, shaping the preference for coiling over clipping for patients with SAH. ${ }^{23,24}$ Since then, many studies comparing interventions in both ruptured and unruptured aneurysms have found that CACo is associated with more favorable outcomes and lower mortality rates than CAC, $13,15,18,20,22-24,32$ including in the elderly population..$^{8,29,32}$ However, other studies have found equivocal results between coiling and clipping. ${ }^{4,18}$ A limited ability to conduct randomized controlled studies in patients with unruptured aneurysms hinders recommendations supported by a high level of evidence. . $^{, 10,13,15}$ Nonetheless, inclination toward CACo over CAC to treat unruptured aneurysms has dominated. ${ }^{4,32}$

There are many studies assessing factors in CAC versus CACo intervention in unruptured aneurysms in the elderly, but limited literature exists to address short-term costs and outcomes in elderly patients compared to nonelderly patients. 6,7,14,16,21,27 Examining in-hospital outcomes for either intervention can quantify its risk weighed against its value. Our study constructs 2 parallel, retrospective cohorts from the National (Nationwide) Inpatient Sample (NIS) database spanning 2002-2013, to examine costs and in-hospital outcomes of treated unruptured aneurysms by
CAC and CACo intervention, comparing results between elderly and nonelderly adults.

\section{Methods}

The NIS, compiled by the Healthcare Cost and Utilization Project (Agency for Healthcare Research and Quality), was used for the present analysis. The NIS is a publicly available sample of inpatient discharge data from approximately $20 \%$ of all hospitals in the US, excluding federal institutions, rehabilitation centers, long-term nonacute care hospitals, psychiatric hospitals, and dependency treatment centers. It is the largest publicly available inpatient discharge database, and thus offers a high degree of external validity. More information about the NIS can be found at http://www.hcup-us.ahrq.gov/nisoverview.jsp. The Rutgers New Jersey Medical School Institutional Review Board does not consider the use of deidentified data from the NIS to meet the regulatory definition of human subject research provided in 45 CFR 46.102. No approval was required for this NIS analysis.

\section{Data Collection and Extraction}

All cases in the 2002-2013 NIS with a primary International Classification of Diseases, Ninth Revision (ICD-9) diagnosis code of 437.3 (cerebral aneurysm, nonruptured) or 430 (ruptured berry aneurysm/subarachnoid hemorrhage) as well as an ICD-9 procedure code for surgical clipping $(39.52,39.72,39.79)$ or endovascular coiling (39.51) were selected. Cases with an ICD-9 code for both surgical clipping and endovascular coiling were excluded. This search yielded a total of 467,186 inpatient hospitalizations, with $35.2 \%$ being for unruptured and $64.8 \%$ being for ruptured aneurysms. Cases with an ICD-9 diagnosis code for ruptured aneurysm were excluded from the present analysis of unruptured aneurysms. Codes for surgical complications were adapted from Bekelis et al. ${ }^{6}$ All cases were weighted, with cases from 2002 to 2011 weighted with the NIS TRENDWT variable, to account for sampling changes beginning with the 2012 data.

The CAC and CACo cohorts were divided into elderly ( $\geq 65$ years of age at admission) and nonelderly $(<65$ years of age at admission) patients for comparison within the 2 interventions. Demographic data characterized included age at admission, sex, and 6 ethnicity and race categories (Asian or Pacific Islander, black, Hispanic, white, other, and unknown). Comorbidities included alcoholism, congestive heart failure (CHF), chronic obstructive pulmonary disease (COPD), coagulopathy, diabetes, hypertension, obesity, peripheral vascular disorder (PVD), and chronic renal failure. The primary outcomes measured included in-hospital mortality and complications during admission. In-hospital complications examined included stroke, treated hydrocephalus, deep venous thrombosis (DVT), pulmonary embolism (PE), cardiac complications, and acute renal failure (ARF). Secondary outcomes examined included length of stay (LOS) and hospital admission charges.

\section{Statistical Analysis}

Independent Student t-test, cross-tabulation, Pearson's 
chi-square, and 2-sided Fisher's exact test were performed as appropriate. Univariate logistic regression analyses were performed with in-hospital outcomes of mortality and complications as the dependent variables to find crude odds ratios. A multiple regression model was then constructed to adjust for the covariates of sex, race or ethnicity, and comorbidities to isolate the effect of age alone. Variables with missing data for more than $10 \%$ of cases were recoded with a dummy variable to account for the missing data in the logistic regression analysis. Two-tailed $\mathrm{p}$ values and $95 \%$ CIs were used and considered significant at $p<0.05$. IBM SPSS version 22 (IBM Corp.) and GraphPad Prism 7 (GraphPad Prism Software, Inc.) were used for statistical analysis.

\section{Results}

Between 2002 and 2013, a total of 21,595 patients were treated for UIAs. Of these patients, 9684 underwent CAC and 11,911 underwent CACo. Within the CAC cohort, there were 7679 nonelderly and 2005 elderly patients. Within the CACo cohort, there were 8281 nonelderly and 3630 elderly patients (Fig. 1).

\section{Demographic Data}

Among patients receiving CAC, 26\% were elderly, and among those receiving CACo, $43 \%$ were elderly. The mean age $( \pm \mathrm{SD})$ of elderly patients in the CAC group was $69.8 \pm 4.1$ years, and the mean age $( \pm$ SD) of the elderly patients in the CACo group was higher, at $71.4 \pm 5.2$ years. Female patients were predominant across patients receiving both CACo and CAC. The majority of patients in both cohorts and age groups identified as white, followed by black, Hispanic, and Asian or Pacific Islander, although a significant proportion of patients were categorized in an "unknown" race and ethnicity category. A comprehensive representation of demographic data appears in Table 1.

\section{Patient Comorbidities}

Among patients who received CAC, significant differences in the prevalence of comorbidities were found between elderly and nonelderly patients. Compared to nonelderly patients receiving CAC, elderly patients had a significantly higher prevalence of CHF (3.6\% vs $1.6 \%)$, COPD (23.2\% vs $16.4 \%$ ), diabetes (15.3\% vs $9.3 \%)$, hypertension $(71.9 \%$ vs $52.9 \%)$, PVD (5.6\% vs $2.5 \%)$, and chronic renal failure (1.3\% vs $0.7 \%)$. Among elderly patients who received CAC, there was a lower prevalence of alcohol abuse than among nonelderly patients $(2.0 \%$ vs $2.9 \%, p=0.022$ ). No significant difference was found between the prevalence of coagulopathy or obesity in elderly and nonelderly groups receiving CAC.

In the CACo group, significant differences in the prevalence of all comorbidities were found between elderly and nonelderly patients. The elderly subgroup had a lower prevalence of alcohol abuse (1.0\% vs $2.1 \%$ ) and obesity (4.6\% vs $6.5 \%$ ), and a higher prevalence of $\mathrm{CHF}(3.6 \%$ vs $1.3 \%)$, COPD (20.3\% vs $15.1 \%)$, coagulopathy (1.8\% vs $1.3 \%$ ), diabetes ( $15.1 \%$ vs $8.8 \%$ ), PVD (7.4\% vs $3.6 \%$ ), and chronic renal failure (3.5\% vs $1.5 \%$ ) (Table 1$)$.

\section{In-Hospital Mortality and Complications}

To eliminate potential confounding by sex, race or ethnicity, and comorbidities, crude odds ratios were adjusted by multiple logistic regression to measure the effect of el-

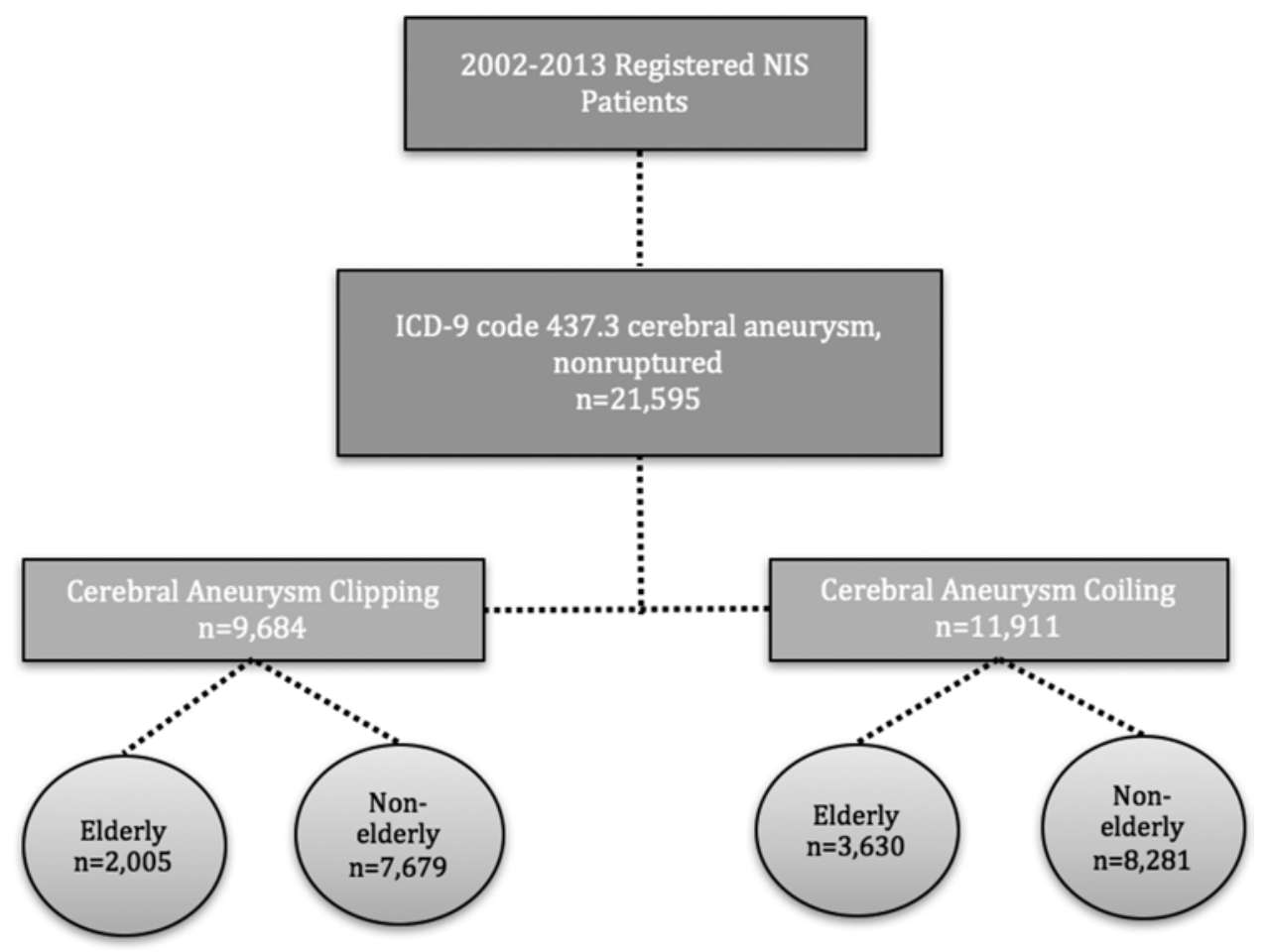

FIG. 1. Algorithm for cohort selection. 
TABLE 1. Demographics and comorbidities in patients with surgically treated UIAs

\begin{tabular}{|c|c|c|c|c|c|c|c|c|}
\hline \multirow[b]{2}{*}{ Variable } & \multicolumn{4}{|c|}{ CAC Group } & \multicolumn{4}{|c|}{ CACo Group } \\
\hline & $\begin{array}{c}\text { Overall } \\
(n=9684)\end{array}$ & $\begin{array}{c}\text { Elderly } \\
(n=2005)\end{array}$ & $\begin{array}{l}\text { Nonelderly } \\
(\mathrm{n}=7679)\end{array}$ & p Value & $\begin{array}{c}\text { Overall } \\
(n=11,911)\end{array}$ & $\begin{array}{c}\text { Elderly } \\
(\mathrm{n}=3630)\end{array}$ & $\begin{array}{c}\text { Nonelderly } \\
(\mathrm{n}=8281)\end{array}$ & p Value \\
\hline Mean age in yrs, \pm SD & $54.3 \pm 11.8$ & $69.8 \pm 4.1$ & $50.3 \pm 9.5$ & $<0.001^{*}$ & $56.9 \pm 12.9$ & $71.4 \pm 5.2$ & $50.6 \pm 9.8$ & $<0.001^{*}$ \\
\hline Sex, $\%$ & & & & $0.008^{*} \dagger$ & & & & $0.231 \dagger$ \\
\hline Male & 25.4 & 23.1 & 26.0 & & 23.4 & 22.7 & 23.8 & \\
\hline Female & 74.6 & 76.9 & 74.0 & & 76.6 & 77.3 & 76.2 & \\
\hline Race, \% & & & & $<0.001^{*} \dagger$ & & & & $<0.001^{*} \dagger$ \\
\hline White & 57.0 & 64.5 & 55.1 & & 57.4 & 64.0 & 54.7 & \\
\hline Black & 8.8 & 4.6 & 9.9 & & 8.3 & 4.6 & 9.9 & \\
\hline Hispanic & 7.3 & 7.2 & 7.3 & & 7.2 & 6.3 & 7.6 & \\
\hline Asian or Pacific Islander & 1.3 & 1.6 & 1.2 & & 1.7 & 2.0 & 1.6 & \\
\hline Other & 2.9 & 3.1 & 2.8 & & 2.9 & 2.6 & 2.1 & \\
\hline Unknown & 22.8 & 19.0 & 23.7 & & 22.5 & 20.5 & 23.2 & \\
\hline Mean LOS in days & 6.6 & 8.0 & 6.3 & $<0.001^{*}$ & 2.9 & 3.2 & 2.8 & $0.002^{*}$ \\
\hline Mean hospital charges in $\$$ & 88,035 & 95,556 & 83,919 & $<0.001^{*}$ & 82,334 & 87,960 & 79,878 & $<0.001^{*}$ \\
\hline Alcohol abuse, $\%$ & 2.7 & 2.0 & 2.9 & $0.022^{*}$ & 1.8 & 1.0 & 2.1 & $<0.001^{*}$ \\
\hline $\mathrm{CHF} \%$ & 2.0 & 3.6 & 1.6 & $<0.001^{*}$ & 2.0 & 3.6 & 1.3 & $<0.001^{*}$ \\
\hline COPD, \% & 17.8 & 23.2 & 16.4 & $<0.001^{*}$ & 16.7 & 20.3 & 15.1 & $<0.001^{*}$ \\
\hline Coagulopathy, \% & 1.8 & 1.9 & 1.7 & 0.709 & 1.4 & 1.8 & 1.3 & $0.021^{*}$ \\
\hline Diabetes, $\%$ & 10.5 & 15.3 & 9.3 & $<0.001^{*}$ & 10.7 & 15.1 & 8.8 & $<0.001^{*}$ \\
\hline Hypertension, \% & 56.8 & 71.9 & 52.9 & $<0.001^{*}$ & 55.6 & 70.8 & 49.1 & $<0.001^{*}$ \\
\hline Obesity, \% & 6.3 & 5.9 & 6.5 & 0.336 & 5.9 & 4.6 & 6.5 & $<0.001^{*}$ \\
\hline PVD, $\%$ & 3.1 & 5.6 & 2.5 & $<0.001^{*}$ & 4.7 & 7.4 & 3.6 & $<0.001^{*}$ \\
\hline Chronic renal failure, $\%$ & 1.9 & 1.3 & 0.7 & $<0.001^{*}$ & 2.1 & 3.5 & 1.5 & $<0.001^{*}$ \\
\hline
\end{tabular}

* Statistically significant.

$\dagger$ Analysis between groups of a given variable (race and sex).

derly versus nonelderly categorical variables alone (Tables 2 and 3, Figs. 2 and 3).

In the CAC cohort, elderly patients had higher perioperative mortality rates $(2.2 \%$ vs $0.8 \%, \mathrm{p}<0.001)$ and greater adjusted odds of in-hospital mortality when compared to the nonelderly group (OR 2.636, p < 0.001). In contrast, among patients receiving $\mathrm{CACo}$, perioperative mortality rates between elderly and nonelderly patients were not significantly different $(0.9 \%$ vs $0.6 \%, \mathrm{p}=0.107)$.
Likewise, the adjusted odds of perioperative mortality did not differ significantly between elderly and nonelderly patients who underwent CACo (adjusted OR 1.364, 95\% CI $0.843-2.208, p=0.206$ ).

Complications in the elderly population with CAC included significantly higher in-hospital rates of stroke $(10.3 \%$ vs $5.8 \%, p<0.001)$, cardiac complication $(2.5 \%$ vs $1.0 \%, \mathrm{p}<0.001)$, and ARF $(1.7 \%$ vs $0.5 \%$, p < 0.001$)$. Elderly patients who received CAC treatments were found

TABLE 2. Complication rates in patients with surgically treated UIAs

\begin{tabular}{|c|c|c|c|c|c|c|c|c|}
\hline \multirow[b]{2}{*}{ Complication } & \multicolumn{4}{|c|}{ CAC Group } & \multicolumn{4}{|c|}{ CACo Group } \\
\hline & $\begin{array}{c}\text { Overall } \\
(n=9684)\end{array}$ & $\begin{array}{c}\text { Elderly } \\
(n=2005)\end{array}$ & $\begin{array}{l}\text { Nonelderly } \\
(\mathrm{n}=7679)\end{array}$ & p Value & $\begin{array}{c}\text { Overall } \\
(n=11,911)\end{array}$ & $\begin{array}{c}\text { Elderly } \\
(\mathrm{n}=3630)\end{array}$ & $\begin{array}{l}\text { Nonelderly } \\
(\mathrm{n}=8281)\end{array}$ & $\mathrm{p}$ Value \\
\hline In-hospital mortality & 1.1 & 2.2 & 0.8 & $<0.001^{*}$ & 0.7 & 0.9 & 0.6 & 0.107 \\
\hline Stroke & 6.7 & 10.3 & 5.8 & $<0.001^{*}$ & 2.8 & 3.5 & 2.6 & $0.005^{*}$ \\
\hline Treated hydrocephalus & 1.9 & 1.9 & 1.9 & 0.898 & 0.8 & 1.0 & 0.7 & $0.042^{*}$ \\
\hline Cardiac complication & 1.3 & 2.5 & 1.0 & $<0.001^{*}$ & 0.4 & 0.7 & 0.3 & $0.001^{*}$ \\
\hline DVT & 0.6 & 0.7 & 0.5 & 0.260 & 0.3 & 0.4 & 0.2 & 0.054 \\
\hline PE & 0.4 & 0.5 & 0.4 & 0.446 & 0.1 & 0.3 & 0.1 & $0.011^{*}$ \\
\hline ARF & 0.8 & 1.7 & 0.5 & $<0.001^{*}$ & 0.6 & 1.0 & 0.5 & $<0.001^{*}$ \\
\hline
\end{tabular}

* Statistically significant. 
TABLE 3. Multiple logistic regression: crude and adjusted odds ratios for in-hospital mortality and complications in elderly versus nonelderly patients after intervention

\begin{tabular}{|c|c|c|c|c|c|c|c|c|}
\hline \multirow[b]{2}{*}{ Complication } & \multicolumn{4}{|c|}{ CAC Group } & \multicolumn{4}{|c|}{ CACo Group } \\
\hline & Unadjusted OR & $p$ Value & Adjusted OR & $p$ Value & Unadjusted OR & $p$ Value & Adjusted OR & p Value \\
\hline In-hospital mortality & 2.704 & $<0.001^{*}$ & 2.636 & $<0.001^{*}$ & 1.447 & 0.109 & 1.364 & 0.206 \\
\hline Stroke & 1.861 & $<0.001^{*}$ & 1.910 & $<0.001^{*}$ & 1.380 & $0.005^{*}$ & 1.381 & $0.007^{*}$ \\
\hline Treated hydrocephalus & 1.024 & 0.898 & 0.995 & 0.979 & 1.540 & $0.043^{*}$ & 1.607 & 0.036 \\
\hline Cardiac complication & 2.429 & $<0.001^{*}$ & 2.195 & $<0.001^{*}$ & 2.490 & $0.002^{*}$ & 2.572 & $0.002^{*}$ \\
\hline DVT & 1.404 & 0.262 & 1.787 & 0.065 & 2.000 & 0.059 & 2.556 & $0.015^{*}$ \\
\hline $\mathrm{PE}$ & 1.322 & 0.447 & 1.345 & 0.441 & 3.810 & $0.010^{*}$ & 3.549 & $0.020^{*}$ \\
\hline ARF & 3.231 & $<0.001^{*}$ & 2.962 & $<0.001^{*}$ & 2.295 & $<0.001^{*}$ & 1.416 & 0.179 \\
\hline
\end{tabular}

Odds ratios (ORs) reflect comparisons between elderly and nonelderly patients. Adjusted ORs represent ORs with corrected values for sex, race, and comorbidities.

* Statistically significant.

to have a 1.910 greater adjusted odds of in-hospital stroke ( $p<0.001$ ), a 2.195 greater adjusted odds of cardiac complication ( $\mathrm{p}<0.001)$, and a 2.962 greater adjusted odds of ARF ( $p<0.001)$. In-hospital rates of treated hydrocephalus, DVT, and PE were not found to have statistically significant differences between elderly and nonelderly groups receiving $\mathrm{CAC}$.

In the CACo cohort, complications in the elderly population also included significantly higher in-hospital rates of stroke (3.5\% vs $2.6 \%, \mathrm{p}=0.005)$, cardiac complication $(0.7 \%$ vs $0.3 \%, \mathrm{p}=0.001)$, and $\mathrm{ARF}(1.0 \%$ vs $0.5 \%, \mathrm{p}<$ $0.001)$. In contrast to the CAC cohort, elderly patients who underwent CACo also experienced statistically higher inhospital rates of treated hydrocephalus $(1.0 \%$ vs $0.7 \%, \mathrm{p}$ $=0.042)$ and PE $(0.3 \%$ vs $0.1 \%, p=0.011)$. The adjust ed odds among the elderly patients who received CACo were greater than in the nonelderly patients for experiencing stroke (adjusted OR 1.381, 95\% CI 1.091-1.747, $\mathrm{p}=$ 0.007 ), cardiac complication (adjusted OR $2.572,95 \%$ CI
$1.408-4.698, \mathrm{p}=0.002)$, DVT $($ adjusted OR $2.556,95 \% \mathrm{CI}$ $1.197-5.460, \mathrm{p}=0.015$ ), PE (adjusted OR $3.549,95 \% \mathrm{CI}$ $1.221-10.313, \mathrm{p}=0.020$ ), and treated hydrocephalus (adjusted OR 1.607, 95\% CI 1.033-2.501, $\mathrm{p}=0.036$ ).

\section{Cost Analysis}

As shown in Table 1, in patients who received either CAC or CACo, the elderly population experienced a longer LOS and incurred higher hospital charges when compared to nonelderly patients. The CAC group had the longest LOS, despite age, with an average LOS of 6.6 days versus 2.9 days in the CACo cohort. The CAC group also had the greatest hospital charges, despite age, with a comparative $\$ 88,035$ to $\$ 82,334$ in the CACo cohort. In the CAC group, the mean LOS among the elderly was 1.7 days longer than the mean LOS among the nonelderly (8.0 vs 6.3 days, $\mathrm{p}<0.001$ ), and the mean hospital charges were $\$ 11,637$ higher among the elderly $(\$ 95,556$ vs $\$ 83,919$, $p$

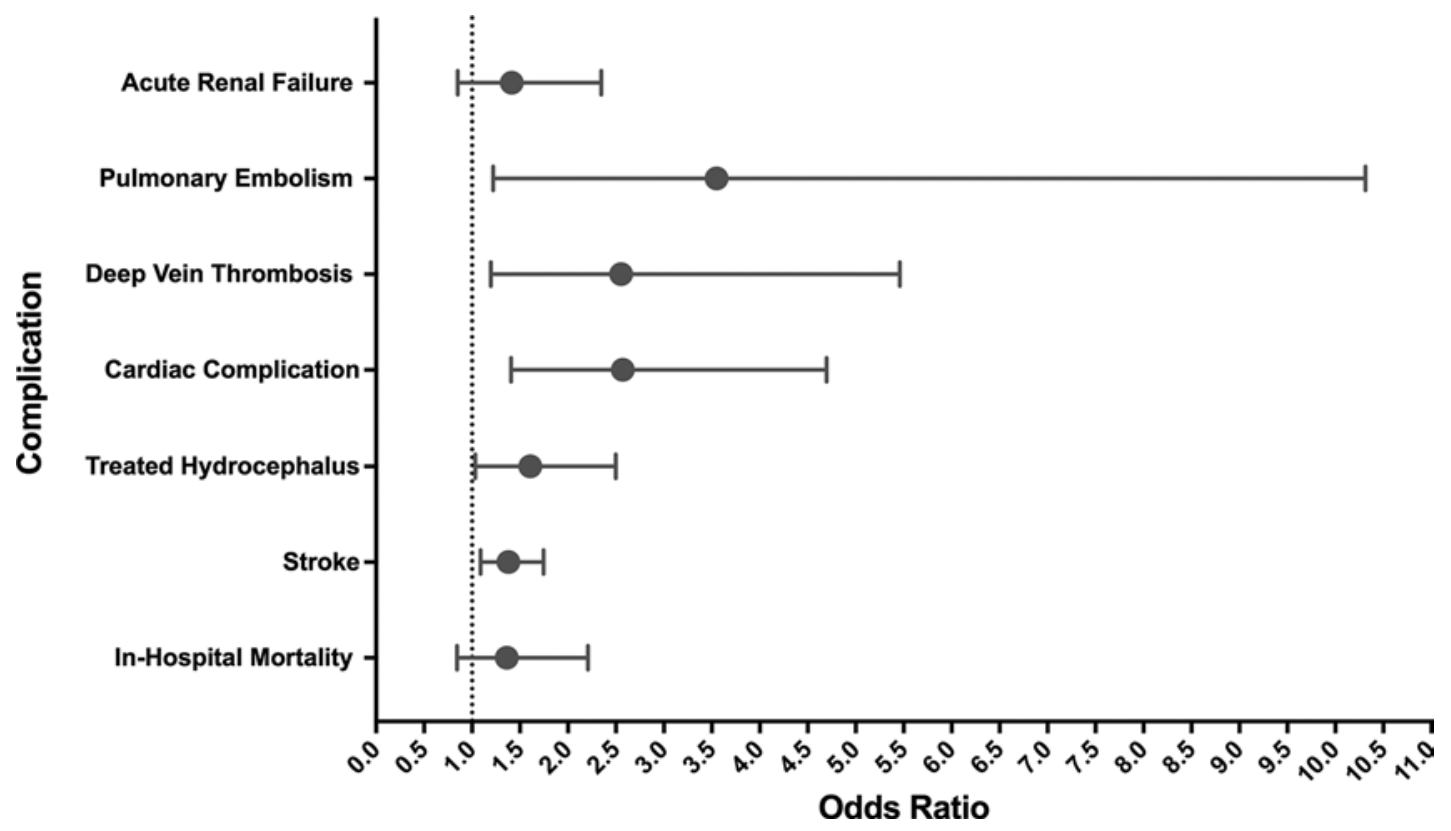

FIG. 2. Adjusted OR with $95 \% \mathrm{Cl}$ of complications following CACo in elderly versus nonelderly patients with UIAs. 


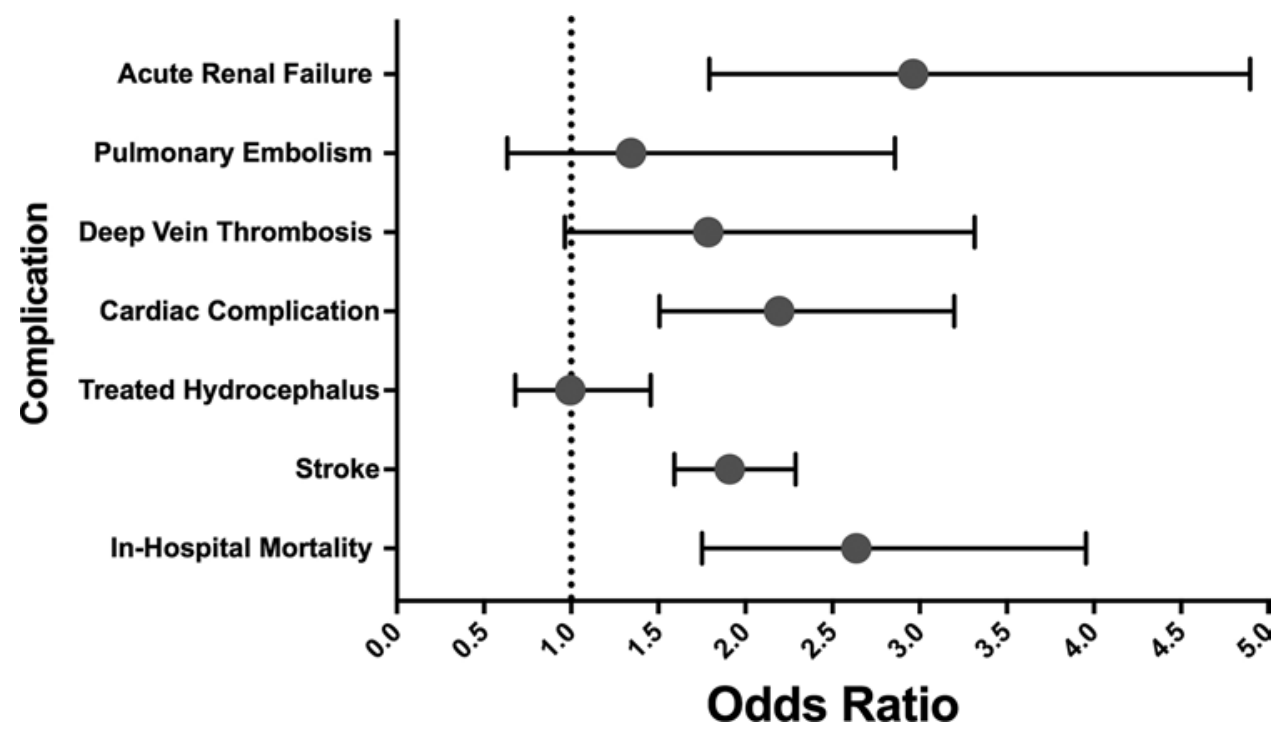

FIG. 3. Adjusted OR with $95 \% \mathrm{Cl}$ of complications following CAC in elderly versus nonelderly patients with UIAs.

$<0.001)$. In the CACo group, the mean LOS among the elderly was 0.40 days longer than the mean LOS among the nonelderly (3.2 vs 2.8 days, $\mathrm{p}=0.002)$, and the mean hospital charges were $\$ 8082$ higher among the elderly (\$87,960 vs $\$ 79,878, \mathrm{p}<0.001)$.

\section{Discussion}

The elderly patient population is of special interest in neurosurgical practice, yet there is a limited evidence base regarding cost-effectiveness and health outcomes in the elderly undergoing intervention for UIA. There are conflicting opinions on when it is appropriate to intervene with $\mathrm{CACO}$ or $\mathrm{CAC}$, rather than continuing conservative management with observation., ${ }^{4,15}$ Among patients undergoing treatment for UIAs, we identified significantly higher mortality and complication rates among elderly inpatients during admission for both surgical clipping and endovascular coiling, although less so in the endovascular coiling group. This was also associated with a longer LOS and higher hospital costs for elderly patients in both treatment groups. These differences remained even after adjustment for comorbidities, sex, and race or ethnicity, demonstrating a higher risk associated with procedural intervention in the elderly population that is independent of their medical comorbidities.

Previous studies have conflicting conclusions on the cost of coiling versus clipping of aneurysms, but they do not focus on the elderly. Hoh et al. ${ }^{17}$ found contrasting results from a single institution, later noting that coiling was more cost-effective than clipping, by using NIS data obtained between 2002 and 2006. Interestingly, clipping was associated with an even longer LOS for unruptured than for ruptured aneurysms, similar to our findings. Halkes et al., ${ }^{14}$ in a European study, found coiling to be more expensive than clipping, solely due to the cost of the coils. Another study concluded that physician reimbursement for coiling was significantly lower than for clipping, yet there was no significant difference between interventions in terms of hospital payments. ${ }^{12}$ Descriptive analysis of the mean hospital charges revealed a high cost of treatment of UIAs in both cohorts. In 2015, Bekelis et al..$^{5}$ reported an average cost of $\$ 35,446$ for CACo between 2005 and 2010 in the NIS database. This is at least half the cost reported by our analysis of both cohorts, indicating an increase in costs over the last decade. The LOS, as would be postulated, was higher in the elderly compared to nonelderly patients in both cohorts, potentially due to the effect of age on comorbidities, complications, and recovery time.

Understanding various health outcomes of either intervention further indicates the most cost-effective method, yet choosing an intervention is determined by multiple variables. Although the use of surgical clipping has decreased with the recent preference for coiling, it is still prevalent in the elderly, as shown in our analysis. In favor of offering CAC in the elderly, Smith et al. ${ }^{29}$ comment on the physiology and aged vasculature of comorbid vessels becoming more difficult to navigate during CACo. Aneurysm size and location also dictate intervention, with middle cerebral artery aneurysms treated with clipping based on better patient outcomes than with coiling. .11,18,27,29,30 Our study demonstrated no significant adjusted odds of ARF in the CACo cohort, although this could be due to pertinent complications of iodine contrast-induced nephropathy in high-risk patients with renal disease. ${ }^{5,26}$ Thus, is it possible that patients with renal disease were preferably treated with CAC. Similarly, the elderly patients who underwent CAC had higher adjusted odds of stroke. As such, the literature has alluded to endovascular procedures correlating with a higher risk of stroke, ${ }^{29}$ thus, it is possible that patients deemed to have a higher risk of stroke preoperatively were chosen to undergo CAC.

Conversely, many studies have deduced that functional outcomes such as quality of life and shorter LOS are more favorable among elderly patients with CACo than in those with CAC.,29 Our analysis found similar outcomes, with significantly higher mortality rates in elderly patients receiving $\mathrm{CAC}$ than in nonelderly patients receiving $\mathrm{CAC}$. 
Interestingly, our study shows that DVT and PE complications in the elderly patients who underwent CACo were substantial. However, elderly patients who received CAC did not have significant odds of these same coagulopathy complications compared to nonelderly patients. Newman et al. ${ }^{26}$ found that coagulopathy as a comorbidity has unfavorable outcomes in treated UIAs. These findings suggest that it should be taken into account during intervention decision making, because patients who undergo CACo could suffer from hypercoagulopathy complications.

With advanced neuroimaging allowing for increased diagnosis and monitoring, some authors have suggested excessive treatment of UIAs in the elderly.,8 The size and location contributes to risk of aneurysm rupture, yet the probability of rupture will vary according to its natural history, with other comorbidities of the elderly more likely to cause death. ${ }^{1,32}$ We agree with Aishima et al. ${ }^{1}$ in that long-term risks of UIAs in the elderly are challenging to determine on the basis of the existing literature. Overall, there is strong evidence that most UIAs are asymptomatic, with a high percentage of incidental findings. ${ }^{25}$ Our findings warrant further analysis for consideration of observation as a safe and cost-effective alternative for the elderly subpopulation. ${ }^{1,2}$ Backes et al. ${ }^{2}$ proposed a risk score instrument for intervention or observation of UIAs, to which we encourage further expansion as an economically useful tactic in decision making.

Although we were able to adjust for comorbidities and demographic variables between our cohorts by using multivariable regression, there was no possible way to adjust for selection bias, such as inherent differences in surgical risk level, location, size, and other natural history variables of aneurysms, because the NIS does not record these variables. Therefore, even methods that attempt to account for covariates that predict receiving a treatment (such as propensity score matching) are not possible. For this reason, we believe that the CACo and CAC groups cannot be directly compared, and only descriptive data can be reported in cost analysis. A recent 2017 analysis of MedPAR (Medicare Provider and Analysis Review) admissions data by Bekelis et al. ${ }^{4}$ does directly compare CACo and CAC of UIAs in the elderly, explaining that these authors' use of instrumental variable analysis adequately adjusts for the inherent selection bias that otherwise renders the 2 groups incomparable..$^{32}$ Our study avoids comparison across differential cohorts by comparing elderly patients to nonelderly patients within each intervention cohort, extracting valuable data on health care outcomes and hospital charges.

\section{Limitations of the Study}

As an NIS database analysis, this study has several important limitations. Coding inaccuracies are inevitable. The NIS does not include useful variables important to the natural history of aneurysmal disease, such as size, location, and morphology of the aneurysm; age of patient at presentation; previous SAH; and family history of aneurysms. Moreover, this study examines cases from the vantage point of a single admission for the purpose of CACo or CAC, and thereby does not include any outcomes data beyond in-hospital data during this admission. Without follow-up data assessing morbidity and mortality in these cases, our study is able to comment on the perioperative management and immediate effects of CACo and CAC, but not on longer-term outcomes. This limits the scope of our study to cost analysis, because cost-effectiveness research requires additional long-term outcome measures in order to compute quality-adjusted life years, or another way of constructing an incremental cost-effectiveness ratio by which our costs can be compared between treatments. ${ }^{33}$ Although it is not feasible to conduct a randomized clinical trial due to the natural history of UIAs, prospective, longitudinal registries studying patients with UIAs are warranted to further understand management of the elderly population in this regard.

\section{Conclusions}

A cost analysis for UIA intervention in the elderly population shows that CAC and CACo are resource intensive and associated with higher risk than in the nonelderly population. CAC in the elderly was found to result in higher in-hospital mortality rates, longer LOSs, and thus, higher mean hospital charges. Candidates for CAC should be managed in light of this association, weighing the risk of death from CAC against the risk of death with continued noninterventional care. CACo or conservative observation, when appropriate, remains a favorable option for treatment of UIAs in the elderly. Further cost-effectiveness research is needed to weigh these costs and outcomes against those of conservative management.

\section{References}

1. Aishima K, Shimizu T, Aihara M, Yoshimoto Y: Lifetime effects of small unruptured intracranial aneurysms. World Neurosurg 95:434-440, 2016

2. Backes D, Rinkel GJE, Greving JP, Velthuis BK, Murayama Y, Takao H, et al: ELAPSS score for prediction of risk of growth of unruptured intracranial aneurysms. Neurology 88:1600-1606, 2017

3. Barker FG II, Amin-Hanjani S, Butler WE, Hoh BL, Rabinov JD, Pryor JC, et al: Age-dependent differences in short-term outcome after surgical or endovascular treatment of unruptured intracranial aneurysms in the United States, 19962000. Neurosurgery 54:18-30, 2004

4. Bekelis K, Gottlieb DJ, Su Y, O'Malley AJ, Labropoulos $\mathrm{N}$, Goodney $\mathrm{P}$, et al: Comparison of clipping and coiling in elderly patients with unruptured cerebral aneurysms. J Neurosurg 126:811-818, 2017

5. Bekelis K, Missios S, Labropoulos N: Cerebral aneurysm coiling: a predictive model of hospitalization cost. J Neurointerv Surg 7:543-548, 2015

6. Bekelis K, Missios S, MacKenzie TA, Desai A, Fischer A, Labropoulos N, et al: Predicting inpatient complications from cerebral aneurysm clipping: the Nationwide Inpatient Sample 2005-2009. J Neurosurg 120:591-598, 2014

7. Brinjikji W, Lanzino G, Rabinstein AA, Kallmes DF, Cloft HJ: Age-related trends in the treatment and outcomes of ruptured cerebral aneurysms: a study of the Nationwide Inpatient Sample 2001-2009. AJNR Am J Neuroradiol 34:1022-1027, 2013

8. Brinjikji W, Rabinstein AA, Lanzino G, Cloft HJ: Racial and ethnic disparities in the treatment of unruptured intracranial aneurysms. A study of the Nationwide Inpatient Sample 2001-2009. Stroke 43:3200-3206, 2012

9. Burnett M, Danish SF, McKhann GM, Le Roux PD: Pathol- 
ogy and pathophysiology of aneurysmal subarachnoid hemorrhage, in Le Roux PD, Winn HR, Newell DW (eds): Management of Cerebral Aneurysms. Philadelphia: Saunders, 2004

10. Cai Y, Spelle L, Wang H, Piotin M, Mounayer C, Vanzin JR, et al: Endovascular treatment of intracranial aneurysms in the elderly: single-center experience in 63 consecutive patients. Neurosurgery 57:1096-1102, 2005

11. Chung RY, Carter BS, Norbash A, Budzik R, Putnam C, Ogilvy CS: Management outcomes for ruptured and unruptured aneurysms in the elderly. Neurosurgery 47:827-833, 2000

12. Drazin D, Dalfino JC, Donovan M, Friedlich D, Feustel PJ, Popp AJ, et al: Surgonomics of unruptured intracranial aneurysms. J Neurointerv Surg 2:168-170, 2010

13. Falk Delgado A, Andersson T, Falk Delgado A: Clinical outcome after surgical clipping or endovascular coiling for cerebral aneurysms: a pragmatic meta-analysis of randomized and non-randomized trials with short- and long-term follow-up. J Neurointerv Surg 9:264-277, 2017

14. Halkes PH, Wermer MJ, Rinkel GJ, Buskens E: Direct costs of surgical clipping and endovascular coiling of unruptured intracranial aneurysms. Cerebrovasc Dis 22:40-45, 2006

15. Hishikawa T, Date I: Unruptured cerebral aneurysms in elderly patients. Neurol Med Chir (Tokyo) 57:247-252, 2017

16. Hishikawa T, Date I, Tokunaga K, Tominari S, Nozaki K, Shiokawa Y, et al: Risk of rupture of unruptured cerebral aneurysms in elderly patients. Neurology 85:1879-1885, 2015

17. Hoh BL, Chi YY, Lawson MF, Mocco J, Barker FG II: Length of stay and total hospital charges of clipping versus coiling for ruptured and unruptured adult cerebral aneurysms in the Nationwide Inpatient Sample database 2002 to 2006. Stroke 41:337-342, 2010

18. Inamasu J, Tanaka T, Sadato A, Hayakawa M, Adachi K, Hayashi T, et al: Outcomes of surgical versus endovascular repair of unruptured brain aneurysms in individuals aged $\geq$ 75 years. Geriatr Gerontol Int 14:858-863, 2014

19. Jang EW, Kim YB, Chung J, Suh SH, Hong CK, Joo JY: Clinical risk factors affecting procedure-related major neurological complications in unruptured intracranial aneurysms. Yonsei Med J 56:987-992, 2015

20. Kawada T, Hishikawa T, Date I, Tominari S, Morita A: Risk of rupture of unruptured cerebral aneurysms in elderly patients. Neurology 86:1650, 2016

21. Lad SP, Babu R, Rhee MS, Franklin RL, Ugiliweneza B, Hodes J, et al: Long-term economic impact of coiling vs clipping for unruptured intracranial aneurysms. Neurosurgery 72:1000-1013, 2013

22. McDonald JS, McDonald RJ, Fan J, Kallmes DF, Lanzino G, Cloft HJ: Comparative effectiveness of unruptured cerebral aneurysm therapies: propensity score analysis of clipping versus coiling. Stroke 44:988-994, 2013

23. Molyneux A, Kerr R, Stratton I, Sandercock P, Clarke M, Shrimpton J, et al: International Subarachnoid Aneurysm Trial (ISAT) of neurosurgical clipping versus endovascular coiling in 2143 patients with ruptured intracranial aneurysms: a randomised trial. Lancet 360:1267-1274, 2002

24. Molyneux AJ, Birks J, Clarke A, Sneade M, Kerr RS: The durability of endovascular coiling versus neurosurgical clipping of ruptured cerebral aneurysms: 18 year follow-up of the UK cohort of the International Subarachnoid Aneurysm Trial (ISAT). Lancet 385:691-697, 2015

25. Morita A, Kirino T, Hashi K, Aoki N, Fukuhara S, Hashimoto N, et al: The natural course of unruptured cerebral aneurysms in a Japanese cohort. N Engl J Med 366:2474-2482, 2012

26. Newman WC, Neal DW, Hoh BL: A new comorbidities index for risk stratification for treatment of unruptured cerebral aneurysms. J Neurosurg 125:713-719, 2016

27. Ryttlefors M, Enblad P, Kerr RS, Molyneux AJ: International subarachnoid aneurysm trial of neurosurgical clipping versus endovascular coiling: subgroup analysis of 278 elderly patients. Stroke 39:2720-2726, 2008

28. Sedat J, Dib M, Lonjon M, Litrico S, Von Langsdorf D, Fontaine D, et al: Endovascular treatment of ruptured intracranial aneurysms in patients aged 65 years and older: follow-up of 52 patients after 1 year. Stroke 33:2620-2625, 2002

29. Smith MJ, Sanborn MR, Lewis DJ, Faught RW, Vakhshori V, Stein SC: Elderly patients with intracranial aneurysms have higher quality of life after coil embolization: a decision analysis. J Neurointerv Surg 7:898-904, 2015

30. Steklacova A, Bradac O, Charvat F, De Lacy P, Benes V: "Clip first" policy in management of intracranial MCA aneurysms: single-centre experience with a systematic review of literature. Acta Neurochir (Wien) 158:533-546, 2016

31. Sturiale CL, Brinjikji W, Murad MH, Lanzino G: Endovascular treatment of intracranial aneurysms in elderly patients: a systematic review and meta-analysis. Stroke 44:1897-1902, 2013

32. Wiebers DO, Whisnant JP, Huston J III, Meissner I, Brown RD Jr, Piepgras DG, et al: Unruptured intracranial aneurysms: natural history, clinical outcome, and risks of surgical and endovascular treatment. Lancet 362:103-110, 2003

33. Zygourakis CC, Kahn JG: Cost-effectiveness research in neurosurgery. Neurosurg Clin N Am 26:189-196, viii, 2015

\section{Disclosures}

The authors report no conflict of interest concerning the materials or methods used in this study or the findings specified in this paper.

\section{Author Contributions}

Conception and design: Gandhi, Silva, Shao. Acquisition of data: Silva, Sylvester. Analysis and interpretation of data: Silva, Shao, Sylvester. Drafting the article: Silva, Shao. Critically revising the article: Gandhi, Silva, Sylvester, Eloy. Reviewed submitted version of manuscript: Gandhi, Eloy. Approved the final version of the manuscript on behalf of all authors: Gandhi. Statistical analysis: Sylvester. Study supervision: Gandhi.

\section{Correspondence}

Chirag D. Gandhi: Westchester Medical Center, Valhalla, NY. chirag.gandhi@wmchealth.org. 